\title{
Experiments with a new Micro-Balance
}

This content has been downloaded from IOPscience. Please scroll down to see the full text. 1919 Proc. Phys. Soc. London 32209

(http://iopscience.iop.org/1478-7814/32/1/323)

View the table of contents for this issue, or go to the journal homepage for more

Download details:

IP Address: 139.184.14.159

This content was downloaded on 01/10/2015 at 21:07

Please note that terms and conditions apply. 
XXI. Experiments with a new Micro-Balance. By $\mathrm{D}_{\mathrm{R}}$. $\mathrm{H}_{\mathrm{ANS}}$ Pettersson.

RECEIVED APRIL 12, 1920.

(COMmunicated вX A. W. Porter, F.R.S.)

CoNSIDERING the fundamental importance of accurate weighings both for physical and chemical research it is surprising how little progress had been made until about ten years ago with regard to improving the sensibility of weighing balances. A certain number of micro-balances had been constructed by Angström, Salvioni, Nernst and others; but their absolute sensibility rarely exceeded a thousandth part of a milligram. Owing to the very delicate mode of suspension chosen for these instruments they were extremely fragile, so that the maximum load which they could safely carry was comparatively small. Their not very considerable gain in absolute sensibility was therefore compensated by a disproportionate loss in relatvve sensibility, which contributed to make their use very limited. The most widely known of these early micro-balances, the Nernst balance, could only be charged with a few cg., which it weighed to about $0.001 \mathrm{mg}$.

Quite a new departure in the technique of micro-weighings was made in 1911, when Steele and Grant published a description of their remarkable all-quartz micro-balance.* By making the beam, as well as the weights and counterpoise, entirely from fused, non-corrodible, and practically nonhygroscopic silica, they were able to give their instrument a very high constancy of its zero. The mode of balancing it on a minute knife-edge of ground quartz made it susceptible to extremely small changes in weight, which could be counterbalanced and measured with a sufficient degree of accuracy, thanks to an ingenious method of aerostatic compensation, namely by varying the pressure of the air inside the case, so as to change the buoyancy of a small sealed silica bulb of known capacity (the "air-weight") suspended from the balance.

Given a certain dexterity on the part of the operator, these instruments could be put to excellent use, as was amply proved by the brilliant work of Sir William Ramsay and Dr. WhytlawGray on the density of niton and on the atomic weight of radium. $\dagger$ 
In the course of these investigations a tiny gold capsule, after being heated red hot, was found to undergo a curious joss in weight, which it slowly regained on cooling. This effect was first supposed to be due to a liberation of air adsorbed by the metal; and when my work at University College, London, was begun in the autumn of 1911 a closer study of this phenomenon was the subject suggested to me by Sir William Ramsay. For this research the quartz micro-balance was to be used, and I was incidentally to try to improve it, so as to make it stronger and less difficult to work with, by suspending it by metal fibres instead of balancing it on a knife-edge.

The advantages of this arrangement appeared somewhat doubtful, considering the rather imperfect elastic properties of metals. Still the method had to be tried. The technical difficulties of soldering thin Wollaston wires to the platinized silica rods of the beam (an operation carried out under the microscope with the aid of a minute electrical soldering apparatus), proved to be unexpectedly great. Several

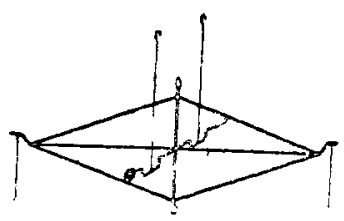

Fig. 1.

months of vain attempts finally resulted in a complete quartz balance suspended by platinum fibres about $0.01 \mathrm{~mm}$. thick. The suspension, however, was entirely unsatisfactory; even at a comparatively low absolute sensibility the zero was found to undergo considerable variations.

Before falling back on the old knife-edge balance $I$ made an attempt to suspend a beam by fine sitica fibres drawn out from the beam itself. The first balance of this type gave very good results, and my subsequent investigations, of which a short account is to be given in the following, have been carried out with similar instruments. The properties of the fibre suspension have been studied theoretically and by experiment. The technique of the instrument itself and of the weighings has been developed. Finally, a fibre-suspended microbalance has been used in some physical investigations. 
It is not possible for me to express adequately my feelings of gratitude to my late teacher, Sir William Ramsay, under whose inspiring influence I have been allowed to work for nearly one year. Probably only those who have themselves had the privilege to work under this "prince de la science" will be able to realise it.

\section{The Fibre Suspendel Micro-Balance.*}

After about ten months spent at University College these investigations were continued in the physical laboratory of Stockholms Högskola, its director, Prof. C. Benedicks, kindly

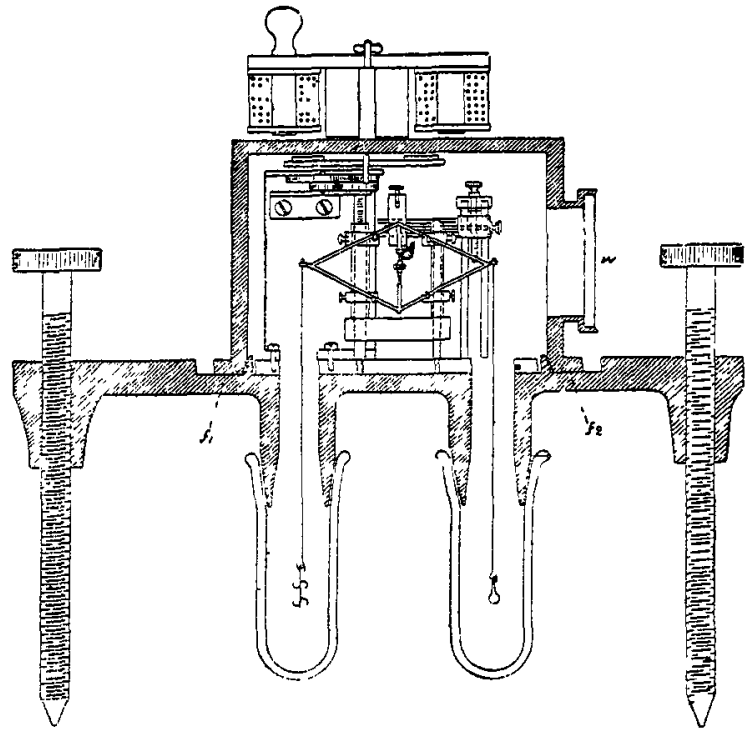

Fig. 2.-Vertical Section of Micro-Balance (to ifalf scale).

putting the necessary instrumental resources at my disposal. With the aid of the skilful mechanic of that institution, Mr. C. Andersson, a new type of case was constructed. Fig. 2 represents a vertical section through a similar case, made for a balance of reduced size by the firm Lyth of Stockholm, who are to manufacture instruments of this hind.

The balance-cases used by Ramsay \& Gray had a solid brass rod, by which the arrestment was worked, ground into

"See my thesis :-_" A New Micro-balance and its use." Goteborg's "Vet.

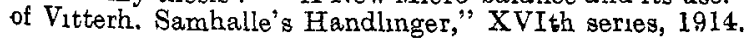


a conical jacket through the floor of the case and made airtight with rubber-grease. On the new case the arrestment is worked without any material transmission, simply by rotating an " electro-magnetic key" on the top of the brass cover, so that a soft-iron anchor-piece is carried round inside by the magnetic forces. In this manner the beam can be lifted off its central fibres when not in use, or made to depend from them when a reading is to be taken.

The supports which grip the silica hooks at the ends of the central fibres can be moved by screws in order to adjust the beam to its correct position, so that the end-fibres depend straight into the centre of the detachable weight-tubes. These are ground to fit over conical brass piec es under the floor of the case, the upper side of which has a circular grove into which the flange $f_{1} f_{2}$ round the cover is ground. All the ground joints are in general made air-tight with rubber grease, a leakage of less than $0.01 \mathrm{~mm}$. of mercury a day being thus obtainable with a vacuum within the case. In certain investigations, where the use of rubber grease was excluded, airtight joints were secured by means of seals made from an alloy of low fusibility, covered externally with low-volatile cement (as a safeguard against porosity of the alloy). Low-volatile cement is also used in case of the plate-glass window, $\mathrm{W}$, through which the light from a Nernst lamp passes to the balance mirror, the image of the filament being focussed on a vertical millimetre scale at a distance of, say, 4 metres.

The shape of the beam itself, as used in my own investigations, is seen from the sketch in Fig. 1. It is made in the ordinary way on the surface of a piece of gas-coal. The fibres are drawn out from small silica-hooks fused to the beam. With a balance of this shape, $10 \mathrm{~cm}$. in length, and weighing about $400 \mathrm{mg}$., a working sensibility of one millionth part of a milligram was realised with a load of $20 \mathrm{mg}$. (i.e., a relative sensibility of one in twenty millions). In one series of measurements a maximum sensibility four times higher was realised, but exact weighings were then made very difficult by the tremors in the Högskola building. These tremors, due to the heavy traffic outside, are rather disconcerting, but did not sensibly affect the balance at moderate values of its sensibility.

With the newest type of fibre-suspended micro-balance of reduced size, having a beam only $5 \mathrm{~cm}$. in length, maximum load. between 100 and $200 \mathrm{mg}$., a maximum working sensibility of 
one ten-millionth part of a milligram is possible, a limit which is desirable for certain investigations.

On the other hand, fibre-suspended balances of a stronger type have also been made, which could weigh 2 grammes to less than $0.001 \mathrm{mg}$. Instruments of this type ought to prove useful for micro-chemical work.

The technique of the weighings with the fibre-suspended balances is similar to that employed with the knife-edge balances, although the operations are considerably simplified owing to improvements in the balance case.

The weighings by pressure are carried out with a bulb of known capacity, $V$, the change in its effective weight due to variation in the pressure and absolute temperature of the air in the case from $p_{1}, T_{1}$, to $p_{2}, T_{2}$ being found from Steele and Grant's equation,

$$
d w=\delta_{0} \frac{273}{760}\left(\frac{p_{1}}{T_{1}}-\frac{p_{2}}{T_{2}}\right) \cdot V
$$

normal density, $\delta_{0}$ of the air being secured by filtering it slowly into the case through tubes containing soda-lime, barium monoxide and dry cotton wool (the last to remove dust particles). The pressure is read on an ordinary mercury manometer in communication with the case; the temperature by a thermometer inserted into a special cylindrical tube sunk into the cover. A quarter of an hour is generally sufficient for the case to take up a uniform temperature.

When changes in weight outside the range of the air-weight are to be measured, one has to make use of silica weights standardised "against air." The lightest of those used by Ramsay \& Gray weighed $0.1 \mathrm{mg}$, and were very difficult to handle. It is preferable to make instead " difference weights" for the fractions of a milligram, i.e., a set of small weights of about $1 \mathrm{mg}$. each, but differing from a certain zero-piece of each set by differences of approximately 1,2,2 and 5-tenths, which differences are also standardised against air.

When working in a vacuum or at a constant pressure small changes in weight can be measured by deflection, whereas larger variations may be counter-balanced by a " magnetic weight " consisting of a small steel needle enclosed in silica, depending from the balance and pulled down by an adjustable magnetic field; this field being produced by sending an electric current through a coil surrounding the weight-tube, and the strength of the current read on a milliampere-meter. The 
value of the "magnetic weight" for different strengths of current has, of course, also to be standardised against air.

The theory of a balance suspended by fibres afiords several points of interest. It is easy to prove that when the beam swings an extremely short length of the fibres becomes curved. This vital part of the fibre needs to be very thin, generally between $1.5 \mu$ and $20 \mu$ for high-sensitivity instruments. The thickness of the rest of the fibres is unimportant. Thin as these fibres are they offer a definite, although very slight, elastic resistance to the bending forces; this increases with increasing deflection of the beam from the upright position, when the fibres are straight. This position consequently represents a maximum of the sensibility, which is reduced on either side of it with growing deflection. This variation, which is relatively small at moderate values of the sensibility, is of little importance as regards the technique of the weighings, since the balance is ordinarily worked at constant deflection. Moreover, as it is perfectly constant, the sensibility curve can easily be determined by means of the air-weight .

From a theoretical point of viow these variations of sensibility are rather interesting, and have been made the subject of a separate study. Their amount decreases with the radius of the fibres as $R^{2}$, whereas the tensile strength of silica fibres is known to decline at a much slower rate; that is, the fibres gain much more rapidly in flexibility than they lose in carrying capacity. One may say, therefore, that the more the size of the instrument is reduced, and the finer the fibres are drawn, the better will this kind of suspension work, whereas the opposite is, of course, the case when knife-edges are used.

The fibre suspension presents several other practical advantages over the knife-edge. The latter is apt to get blunt with use and is easily dismounted; it often gets fouled by dust particles. The fibres retain their elastic properties indefinitely and cannot be fouled in any way.

At ordinary pressures the swing of the balance is strongly damped by air friction, but in certain experiments in a charcoal vacuum the beam was found to keep up its swinging motion without any sensible damping, the energy dissipated within the thin fibres being of course infinitesimal. Owing to the perfect properties of this suspension it should be possible to increase the accuracy of the weighings simply by reading the position of the balance very sharply, say with a microscope or 
by making use of increased scale distance. The sensibility curve at the end of this Paper seems to confirm this view.

\section{Investigations with the Micro-Balance.}

Of the numerous subjects of research with the micro-balance which have suggested themselves to the author, only a few have hitherto been carried out, mainly owing to the pressure of other work.

By weighing in a charcoal vacuum a small sphere of solid silica depending from the balance into a long silica tube, which could be heated up to $1,000^{\circ} \mathrm{C}$. by a cylindrical electric furnace, it has been possible to repeat on a diminutive scale and at much higher temperature the experiments of Poynting \& Phillips on the influence of temperature upon weight. The upper limit for any possible temperature coefficient of gravity $\left(10^{-9}\right.$ per $1^{\circ} \mathrm{C}$.) found by these authors within the range $-180^{\circ} \mathrm{C}$. to $100^{\circ} \mathrm{C}$, was found to hold up to $460^{\circ} \mathrm{C}$. with rather a higher degree of accuracy. When the experiments were repeated at $600^{\circ} \mathrm{C}$, the silica sphere was found to undergo a gradual loss in weight at an approximately constant rate, which was much increased when the temperature was raised to nearly $800^{\circ}$. This rather unexpected result indicates that in a high vacuum silica is sensibly volatile even at $1,000^{\circ} \mathrm{C}$. below its melting point! This substance cannot therefore be used for an extension of these experiments to still higher temperatures.

A similar or slightly modified experimental arrangement can obviously be used for investigations regarding the volatility of various substances at relatively low temperatures, and also for exact measurements of their vapour tension.

The changes in weight of a piece of gold subsequent to heating, which Ramsay and Gray had discovered and explained as due to adsorbed air, has also been studied. For this purpose a minute electric furnace (made from platinum leaf over a framework of silica) was mounted within the weighttube, so that a plece of gold foil surrounded by the furnace could be heated at different pressures while suspended from the balance, and weighed immediately afterwards. The results proved that an adsorption and liberation of air from the gold could not be the cause of the phenomenon, but that this was due to a condensation on the metallic surface of vapours from the rubber grease used to make the case airtight. Although the effect thus loses some of its theoretical 
interest, it is of a certain importance for the technique of the weighings, and will be made the subject of further research.

A subject which has hitherto been practically inaccessible to experimental work owing to the lack of sufficiently sensitive balances is the magnetic suaceptibility of diamagnetic gases and vapours. A silica micro-balance affords the most favourable possibilities for such measurements, and it has also been used for determining the magnetic susceptibility of pure nitrogen and hydrogen gas. The former substance does not

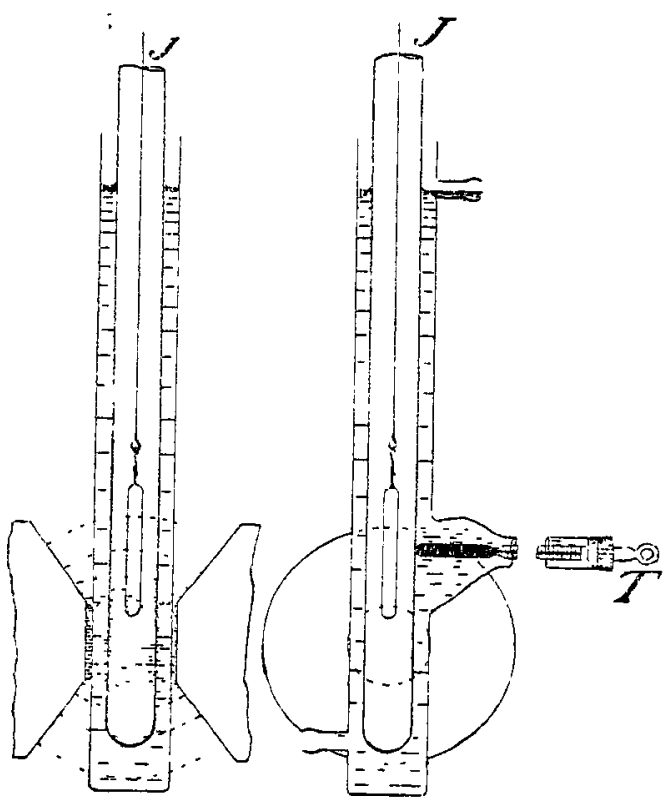

FIG. 3.

seem to have been at all studied by direct experiments, whereas the most recent measurement with pure hydrogen (made by Bernstein in 1909) gave a result about 100 per cent. higher than the correct value.*

The method employed is made clear by the sketch in figure 3 , which represents a very light and narrow cylindrical bulb depending from the balance, with its lower end near the centre of a strongly divergent magnetic field.

* Bernstein : "Inaugural dissertation." 
The "magnetic broyancy" due to the gas round the bulb when the field was applied was compensated by varying the pressure. Experiments were made with air (at different pressures) and with very pure nitrogen and hydrogen gas, so that the magnetic susceptibility of the latter could be calculated from the known susceptibility of air. The results agree well with the theoretical values calculated by M. Pascal from the diamagnetism of nitrogen and hydrogen compounds, viz. - *

hydrogen $\kappa=-2 \cdot 62 \times 10^{-10}$ (theoretical value $\left.\kappa=-2 \cdot 6 \times 10^{-10}\right)$ nitrogen $\kappa=-5.57 \times 10^{-10}$ (theoretical value $\kappa=-5.0 \times 10^{-10}$ )

The susceptibility of liquid hydrogen has been measured by Kamerlingh-Onnes \& Perrier, the result recalculated for hydrogen gas giving $\kappa=-2 \cdot 4 \times 10^{-10} . \dagger$

The sensibility of the balance in itself, although moderate, would have allowed of an accuracy of about 1 per cent. That the probable error in the above results is nearly ten times larger is chiefly due to the large errors caused by temperature variations. In order to compensate for the diamagnetism of the silica in the bulb the latter had been filled with a suitable mixture of air and oxygen, so as to make it magnetically neutral at room temperature. The paramagnetism of the enclosed oxygen being a function of the temperature, this arrangement was highly susceptible to temperature changes, which could not be entirely eliminated $b y$ the water-jacket shown in the sketch. With a similar experimental arrangement modified so as to neutralise the temperature effect, the magnetic properties of other gases and vapours might be accurately measured. The author hopes especially to investigate whether when absorbing or fluorescing under the influence of light a gas or vapour has its magnetic susceptibility affected.

There are, besides the subjects mentioned, a great number of others calling for investigation with the micro-balance. Only a few of these will be here indicated.

The experiments of Nichols \& Hull $\ddagger$ on the pressure of light might be repeated with a much simpler arrangement, by weighing on a highly sensitive micro-balance the pressure exerted on a reflecting disc from below by an ascending beam of light. An accuracy of measurement of 1 per cent. or even

"Pascal : "Ann. Chim. Phys.," VIII., 19, p. 5.

+ Kammerlingh-Onnes and Perrier:" Comm. Phys. Lab. Leyden," 122 A., p. 10 .

† Nichols \& Hull : “Drude's Ann.," 12, p. 225. 
less would be quite feasible, and would make possible interesting variations in the conditions of the experiment.

The ingenious method due to Prof. Knudsen* for measuring accurately very low pressures by an "absolute manometer" (by measuring the radiometric force between two discs of known unequal temperatures in a gas at low pressure) might be applied to the measurement of absolute temperatures with the aid of the micro-balance. If two horizontal discs of unequal temperatures $T_{1}$ and $T_{2}$, one of them suspended from the balance, are brought within suitable distance of each other, the radiometric force $F$ between them could easily be determined by weighing. Supposing the pressure $p$ of the gas in the case (say highly rarenied helium) to be accurately known, then the ratio between the absolute temperatures is given by Knudsen's equation $F=\frac{p}{2} \sqrt{\frac{I_{1}-T_{2}}{T_{2}}}$,

and if either temperature is known the other can be calculated. By this method temperatures near absolute zero might be measured with a high degree of accuracy.

According to modern conceptions of the relationship between energy and matter the gain or loss of a certain amount of energy, $d E$, from a body should correspond to a change in its weight, $d W$, given by $d W=\frac{d E}{c^{2}}$, where $c$ is the velocity of light. All ordinary changes in energy are too small to give rise to any perceptible changes in weight according to this formula, the only exception being those accompanying radioactive disintegration. If a large quantity of radium emanation, say, 2 curies, could be weighed, first fresh and then after disintegration into Radium $D$, the "weight" of the energy given off in the meanwhile should be of the order of one millionth of a milligramme, an amount which might be measured with a highly sensitive micro-balance. Owing to the intense thermal and electric phenomena accompanying the disintegration, the technical difficulties of making such weighings will no doubt be formidable, but the importance of such an "experimentum crucis" appears so considerable as to make it well worth an attempt.

\section{The Stromberg Suspension.}

The number of investigations open to micro-balances, which would add at least three or four decimals to the ordinary range

* Knudsen: "Ann. der Phys.," 28, p. 75. 
of measurement, with a gain instead of a lose in relative sensibility, is obviously almost unlimited.

It is therefore rather remarkable that, except for the work of Ramsay and Gray, the quartz micro-balance remains almost unused even to-day. The reason is probably that it appears to be regarded as extremely difficult to work with, and requiring a quite unusual manipulative skill on the part of the operator. This impression had some foundation as regards the knife-edge balances and also, although to a less degree, regarding the early types of the fibre-suspended balances. Thanks to some very important improvements in construction

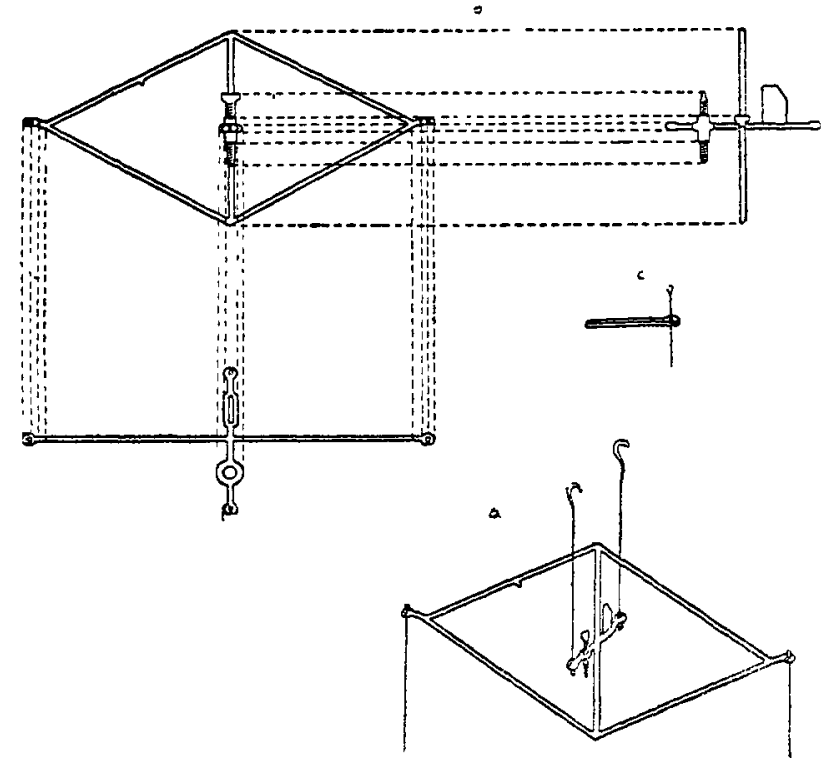

í. 4.-The Strömberg Suspension.

which have lately been worked out at Stockholms Högskola, by Dr. Stromberg, these are now quite as simple to work with as any other highly sensitive physical instrument.

The Stromberg suspension is sketched in Fig. 4, and needs but a few words of explanation. The fibres are made detachable, with small conical lumps at one end which fit into small silica rings on the beam. The fine part of the fibre is passed through a narrow cut in the side of the ring, and the conical lump is secured in its place with a minute quantity of shellac. If a fibre should be accidentally broken a reserve fibre can be 
inserted in its place in a few minutes without removing the balance from its place.

In the early instruments where the fibres were drawn out from the beam, a broken central fibre could only be repaired by dismounting and inverting the beam and drawing out from it a new fibre, an operation which was rather delicate and not without a certain risk of destroying one of the other fibres. Another improvement of considerable practical value, which has also been worked out by Dr. Strömberg, is the tiny magnalium screw seen in the figure, by which the centre of gravity of the beam (and consequently also its sensibility) can be raised without difficulty. The older method of sealing on to the beam, or drawing off from it minute pieces of silica, was dangerous to the fibres, and also tedious as it was done quite at random, and therefore had to be repeated a great number of times in order to realise the desired adjutment.

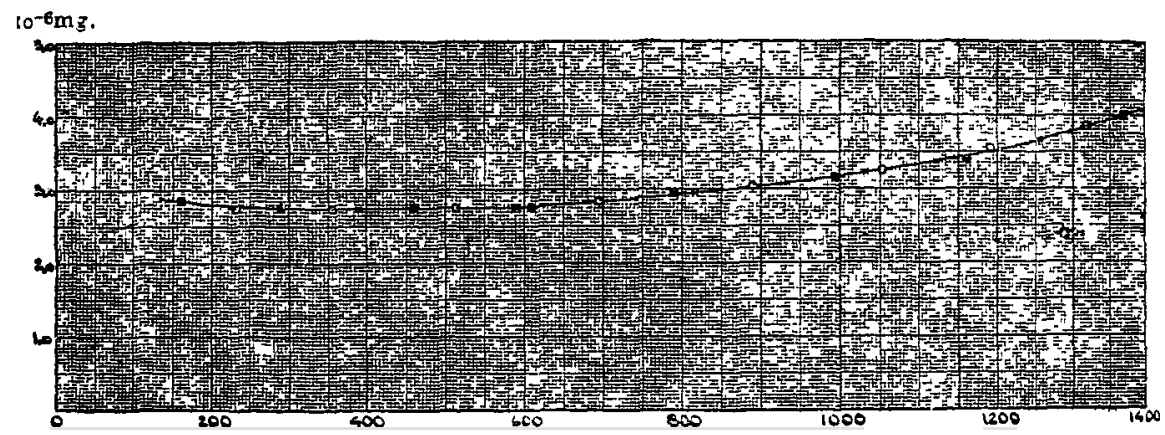

FIG. 5.

With the permission of Dr. Strömberg I am able to reproduce in Fig. 5 a sensibility curve obtained with one of his instruments (which I had the pleasure of showing to the Physical Society of London at its meeting on March 12, 1920). It is derived from three independent series of measurements, and although the scale-sensibilty is very moderate $\left(2.7 \times 10^{-6} \mathrm{mg}\right.$. per $1 \mathrm{~mm}$. scale-deflection at four metres scale-distance), corresponding to a relatively short period of swing, the different points on the curve are seen to agree to within less than one ten-millionth part of a mg. This result lends support to the view that the suspension of the instrument is in itself sufficiently delicate to allow the working sensibility to be considerably raised, merely by increasing the accuracy with 
which the position of the balance is observed, without any further prolongation of the period of swing.

Thanks to these improvements made by Dr. Strömberg, who will shortly publish an account of his own work, the technique of the fibre-suspended micro-balance has been much simplified, and it can now be used by anybody with scientific training. Without exaggeration one may, therefore, say that this micro-balance, which in its origin is due to the genius of British men of science, to Mr. C. V. Boys, who first invented the wonderful quartz-fibres, to Steele and Grant, to Ramsay and Gray, has by stages been developed into a working instrument which provides the chemical and physical sciences with a new and powerful weapon for future research.

\section{ABSTRACT.}

Previous Micro-balances.-Brief reference is made to the instruments of Angström, Salvioni, Nernst, and especially to the all-quartz balance of Steele and Grant.

The Author's Micro-balance.-The instrument devised by the author, and here described, is also wholly of quartz. The knife-edge support is, however, replaced by a suspension consisting of a pair of quartz fibres. A magnetic arrestment is employed. Balance is obtained either (a) by an "air-weight," the upward displacement on a bulb being varied by varying the gas pressure within the enclosing case ; or $(b)$ by a "magnetic weight," involving the use of a measured electric current. The range of weighings depends on the size of beam : with one $5 \mathrm{~cm}$. in length the maximum load is between 100 and $200 \mathrm{mg}$., and the setting can be made to within $10^{-7} \mathrm{mg}$.

The Stromberg Suspension.-Improvements in the construction devised by Dr. Strimberg are described, which obviate the formidable difficulties of manipulation that have hitherto discouraged workers from employing the instrument.

Investigations with the fibre-suspended Micro-balance.-A brief description is given of the following researches already carried out:-(1) The influence of temperature upon weight. This is an an extension of the work of Poynting and Phillips on this subject; (2) the volatility of silica ; and (3) the susceptibility of diamagnetic bodies.

It is proposed to conduct investigations on the pressure of light. on the measurement of absolute temperature (based on an equation of Knudsen's), and on the loss of mass accompanying loss of energy. 